\title{
Effect of an ankle-foot orthosis on gait kinematics and kinetics: case study of post-stroke gait using a musculoskeletal model and an orthosis model
}

\author{
Masataka Yamamoto ${ }^{*}$, Koji Shimatani $^{2}$, Masaki Hasegawa ${ }^{2}$ and Yuichi Kurita ${ }^{1,3}$
}

\begin{abstract}
One of the recommended post-stroke gait rehabilitation treatments is the use of an ankle-foot orthosis. In clinical practice, it is important to adjust the torque of the ankle-foot orthosis assistance to suit each patient's body function and gait ability. The present study aimed to investigate the effect of changing the plantar flexion resistance of the ankle-foot orthosis on the post-stroke gait kinematics and kinetics during the early stance phase using a musculoskeletal model and an ankle-foot orthosis model. The subject was a male with post-stroke left hemiplegia who could walk independently without an ankle-foot orthosis and/or cane. The subject walked at a self-selected speed under the no ankle-foot orthosis condition and three ankle-foot orthosis conditions, each with a different plantar flexion resistive torque. A motion analysis system was used to measure the following spatiotemporal parameters: gait speed, step length, cadence, and step length ratio. In addition, the ankle angle of the paretic side, ankle torque of the paretic side, and plantar flexion resistance torque of the ankle-foot orthosis were calculated using a musculoskeletal model and an ankle-foot orthosis model. The results showed that the gait speed and step length ratio of all ankle-foot orthosis conditions were improved compared with the no ankle-foot orthosis condition. In particular, the condition with the smallest torque was the most symmetric of the four walking conditions. The condition with the smallest torque also resulted in the greatest increase in the dorsiflexion angle of the paretic side at heel contact. The internal dorsiflexion torque was most increased in the ankle-foot orthosis condition with the smallest torque for this subject. The simulation of the post-stroke gait in the present study contributes to the development of more effective gait rehabilitation treatment methods using an ankle-foot orthosis.
\end{abstract}

Keywords: Simulation, Ankle foot orthosis, Stroke, Gait

\section{Background}

The main causes of reduced gait function are disease and aging. Stroke is a typical disease that causes gait disability due to hemiplegia and sensory disorder. Stroke patients generally have decreased gait speed and step length, with an asymmetrical and abnormal gait pattern $[1,2]$. In addition, post-stroke walking is made difficult by abnormal gait patterns such as drop foot and instability

\footnotetext{
*Correspondence: masatakayamamoto@hiroshima-u.ac.jp

${ }^{1}$ Graduate School of Engineering, Hiroshima University, 1-4-1,

Kagamiyama, Higashi-Hiroshima City, Hiroshima 739-8527, Japan

Full list of author information is available at the end of the article
}

in the stance phase. Drop foot comprises difficulty in performing dorsiflexion, causing decreased toe clearance in the swing phase and forward propulsion in the stance phase, both of which increase the risk of falling. Thus, it is important to improve the post-stroke gait pattern to improve safety during walking.

An ankle-foot orthosis (AFO) is one of the methods recommended for the treatment of post-stroke gait abnormalities. Several studies have reported that using an AFO on the paretic side improves gait speed, balance, energy cost, and gait kinematics [3-5]. Although the AFO has several functions, the plantar flexion resistance (PFR) function is effective in improving the post-stroke 
gait. The PFR generates torque to resist ankle plantar flexion and it assists coordinated movement of ankle joint instead of dorsiflexion torque. It is difficult for people with post-stroke to create dorsiflexion torque because they can not control ankle motion and create torque coordinately. Although dorsiflexion torque in early stance is small, this torque is important to create forward propulsion and absorb shocks [6]. Therefore, the PFR of AFO not only improves drop foot, but also improves the rapid ankle plantar flexion in the early stance phase from heel contact to the loading response. An AFO with PFR improves the weight acceptance response in the early stance phase, which positively influences gait function $[7,8]$. However, the effect of the magnitude of the PFR torque on gait kinematics and kinetics remains unclear.

Musculoskeletal model simulations can be used to estimate the effect of an assistive device on human movement. Murai et al. [9] used multilayered kinodynamics simulation to analyze the joint torque of amputees with prostheses during sprinting. Other previous model simulation studies have evaluated the effect of substituting an AFO with resistive moment for the function of the soleus muscle during walking in healthy subjects [10], and analyzed whether an AFO with dorsiflexion resistance moment affects the Achilles tendon function during walking in healthy subjects [11]. Our previous studies have revealed that variations in the PFR affect the muscle force and joint reaction force during gait in healthy adults $[12,13]$. However, the contribution of the magnitude of the torque produced by an AFO on post-stroke gait is unclear. Although many papers reported about net joint moment, the paper that reported joint torque and AFO torque in stance phase separately were few. It is also important to understand how changed not net joint moment but internal joint moment for more effective treatment.

So, the purpose of the present study investigated the effect of the magnitude of the PFR of an AFO on poststroke gait kinematics and kinetics during the early stance phase using a musculoskeletal model and an AFO model. In addition, we investigated joint torque and AFO torque in stance phase separately. This post-stroke gait simulation findings will contribute to the development of more effective gait rehabilitation using an AFO.

\section{Methods}

\section{Subject}

The subject was a male with post-stroke left hemiplegia resulting from a stroke that had occurred more than 6 months previously. Despite the mild hemiplegia, the subject could walk independently without an AFO and/ or cane. The subject did not have limitation of activity due to heart disease. Table 1 shows the subject's clinical characteristics.

\section{AFO conditions}

An articulated AFO with an adjustable PFR function (Gait Solution Design, Kawamura Gishi, Osaka, Japan) was used in the present study (Fig. 1). The AFO comprised a footplate and a cuff, and generated a PFR torque without a dorsiflexion resistive torque. The PFR could be adjusted from a setting of 1 (very flexible) to 4 (rigid) [14]. In our study, the PFR was set to be generated when the ankle angle of the paretic side was less than $5^{\circ}$ of dorsiflexion.

\section{Measurement protocol}

The subject walked under four conditions: no AFO, PFR1 (PFR, $0.56 \mathrm{Nm} /{ }^{\circ}$ ), PFR2 (PFR, $0.76 \mathrm{Nm} /{ }^{\circ}$ ), and PFR3 (PFR, $0.95 \mathrm{Nm} /{ }^{\circ}$ ). We did not set PFR4 condition because further increases in PFR caused an increase in gait abnormality and walking difficulty due to excessive AFO torque. After sufficient walking practice, the subject walked on a $10 \mathrm{~m}$ walkway at a self-selected, comfortable speed under each of the four conditions. Walking in each condition was repeated until three successful trials that one gait cycle measured completely. The no AFO condition was measured first, followed by the three PFR conditions. The order of the PFR conditions was randomized. The subject wore the AFO on the left foot, which was the paretic side, and wore the same type of shoe on both feet.

Gait data were measured using the VICON MX motion analysis system (Vicon, Oxford, UK). This system included 12 infrared cameras (sampling rate, $100 \mathrm{~Hz}$ ) and six force plates (AMTI, Watterson, MA, USA; Kistler, Amherst, NY, USA) (sampling rate, $1000 \mathrm{~Hz}$ ). For motion capture, the reflective markers were affixed over the following anatomical landmarks: seventh cervical vertebra, sternoclavicular notch, xiphoid process, right scapular inferior angle, and tenth thoracic vertebra. Additionally,

\section{Table 1 Clinical characteristics of the subject}

\begin{tabular}{ll}
\hline Parameters & Value and name \\
\hline Age (years) & 77 \\
Height $(\mathrm{cm})$ & 163.4 \\
Weight $(\mathrm{kg})$ & 67.5 \\
Diagnosis & Cerebral hemorrhage \\
Paretic side & Left side \\
Brunnstrom recovery stage (lower extremity) & $\mathrm{V}$ \\
Sensory disorder & Mild \\
Modified ash worth scale of ankle joint muscles & 0 \\
Range of ankle joint motion contracture & None \\
\hline
\end{tabular}




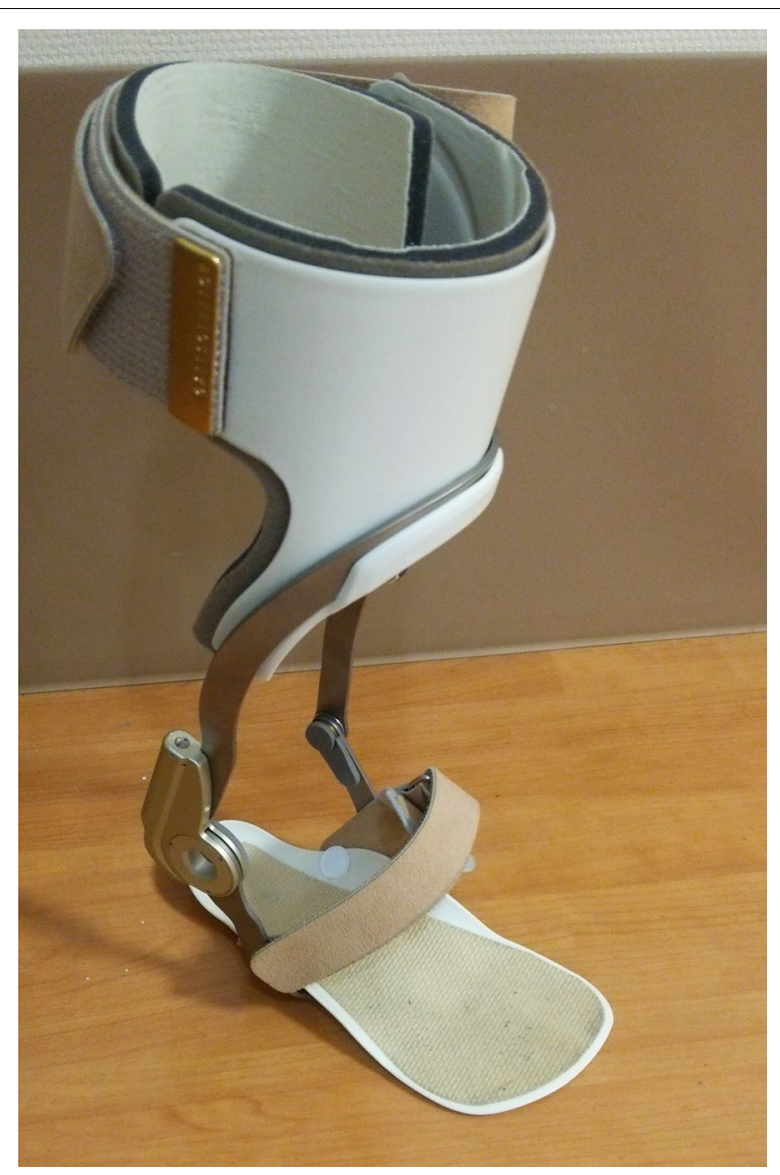

Fig. 1 The ankle foot orthosis was used in present study

the markers were affixed bilaterally over the anterior and posterior acromion processes, anterior-superior iliac spines, posterior-superior iliac spines, lateral thighs, medial and lateral epicondyles of the femurs, lateral shanks, medial and lateral malleoli, calcanei, heads of the second and fifth metatarsals, and tips of the second toes. In the PFR conditions, the left side medial and lateral malleoli markers were placed directly on the AFO so that they were visible.

\section{Musculoskeletal model and AFO model}

The three-dimensional coordinates of the marker data and ground reaction force data provided by the motion analysis system were used for simulations using a musculoskeletal model and an AFO model (Fig. 2). OpenSim, an open-source musculoskeletal modeling and simulation software platform, was used to calculate the kinematics and kinetics during gait [15]. The present study used the gait2392 musculoskeletal model, which has 23 degrees of freedom. The AFO model of the ToyLanding model was connected with the musculoskeletal model [16]. These models were the same as those used in our previous study [13]. The AFO model comprised a footplate and cuff that were rigidly attached to the left foot and tibia, respectively. The footplate and cuff were connected at two hinge points, allowing both dorsiflexion and plantar flexion movements at the ankle joint. Both the resistance and the ankle angle at which resistance was exerted in the AFO were adjusted to create each PFR condition. The PFR was set to be generated when the ankle dorsiflexion was less than $5^{\circ}$. We measured the experimental torque value in each PFR condition using a Gait Judge System which include a torque sensor (Kawamura Gishi, Osaka, Japan), and inputted the data into the AFO model. Therefore, PFR was just the torque of AFO that generated against plantar flexion movement, and it did not include muscle force.

We performed the following procedure to calculate the ankle joint angle and internal joint torque. The musculoskeletal model was scaled to match the anatomical landmarks of the subject using the scale tool in OpenSim. The dimensions of each body segment of this model were scaled based on the relative distances between pairs of reflective markers that were measured by the motioncapture system and the corresponding virtual marker locations during a static trial [17]. Then, the ankle joint angle of the paretic side was estimated using inverse kinematics. The use of inverse kinematics minimized the errors of the trajectories between the experimental markers of the motion capture system versus the virtual markers of the scaled model. Finally, we calculated the internal ankle joint torque of the paretic side using inverse dynamics. Inverse dynamics used the motion of the musculoskeletal model to solve the equation of motion for the unknown vector of generalized forces and torques $\boldsymbol{\tau}$. In accordance with the previous studies [18, $19]$, the equation was expressed as

$$
\boldsymbol{\tau}=\boldsymbol{M}(\boldsymbol{q}) \ddot{\boldsymbol{q}}+\boldsymbol{C}(\boldsymbol{q}, \dot{\boldsymbol{q}})+\boldsymbol{G}(\boldsymbol{q})
$$

where $\boldsymbol{q}, \dot{\boldsymbol{q}}$, and $\ddot{\boldsymbol{q}}$ were the vectors of generalized positions, velocities, and accelerations, respectively, $\boldsymbol{M}(\boldsymbol{q})$ was the system mass matrix, $C(\boldsymbol{q}, \dot{\boldsymbol{q}})$ was the vector of the Coriolis and centrifugal forces, and $\boldsymbol{G}(\boldsymbol{q})$ was the vector of gravitational forces $[18,19]$.

The equation was used to calculate the net forces and torques at each joint that produced the movement. In case of present study, the net ankle joint torque consists of the internal ankle joint torque and the PFR torque of AFO. So, internal ankle joint torque means the purely moment that generated inside the subject's body. The ankle joint torque of each of the AFO conditions (PFR1, PFR2, and PFR3) was subtracted from each PFR torque during the stance phase to enable the comparison of the internal ankle joint torque alone between the no AFO condition versus the AFO conditions. 


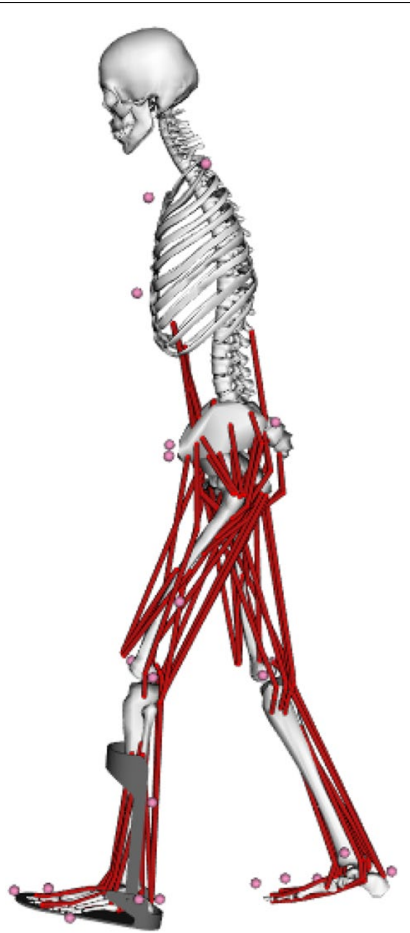

Fig. 2 Musculoskeletal model and ankle foot orthosis model

\section{Data analysis}

The spatiotemporal parameters (gait speed, left and right step lengths, and cadence) in each condition were measured directly from experimental data. The step length ratio was calculated to enable the assessment of gait symmetry. A step length ratio of closer to 1 indicates a more symmetrical gait pattern. The ankle joint angle at heel contact and the minimum dorsiflexion angle in the early stance phase were calculated from the model simulation. Peak internal dorsiflexion torque and PFR torque were also calculated from the model simulation. We focused on the early stance phase because this is the stage during which the PFR affects ankle function and weight acceptance $[7,8]$. These data were averaged and compared among the four walking conditions.

\section{Results}

Spatiotemporal parameters

Table 2 shows the spatiotemporal parameters in each condition. The gait speed tended to be greater in all AFO conditions compared with the no AFO condition. The left side step length tended to increase in the PFR1 and PFR 2 conditions compared with the no AFO condition. However, the left side step length in the PFR3 condition was similar to that of the no AFO condition. The right side step lengths in the PFR1 and PFR3 conditions were similar to that in the no AFO condition. The step length ratio in all PFR conditions tended to indicate a more symmetric gait than that in the no AFO condition. The PFR1 condition produced the most symmetric gait pattern of the four walking conditions.

\section{Ankle angle}

Figure 3 shows the ankle joint angle of the left side in the stance phase, while Fig. 4 shows the left ankle joint angle at heel contact. The ankle joint angles at heel contact for each condition were: no AFO, $4.72^{\circ} \pm 1.29^{\circ}$; PFR1, $6.71^{\circ} \pm 1.45^{\circ}$; PFR2, $6.55^{\circ} \pm 0.04^{\circ} ;$ PFR3, $6.19^{\circ} \pm 0.13^{\circ}$. The ankle joint angles in all AFO conditions tended to be greater than that in the no AFO condition.

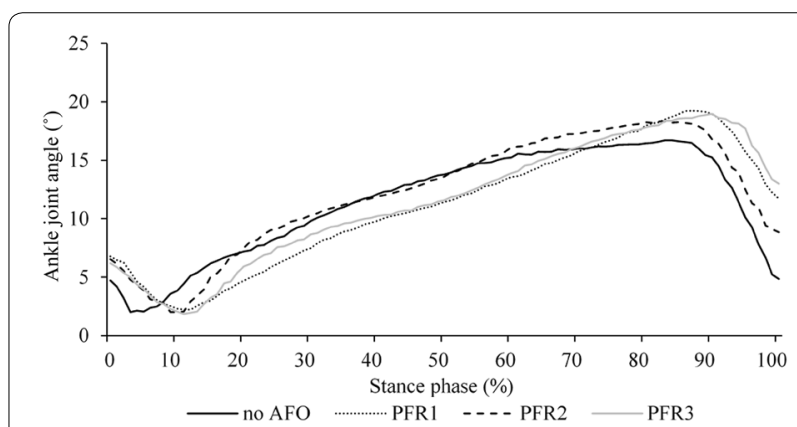

Fig. 3 Ankle joint angle in stance phase. Dorsiflexion is defined as positive. The \% stance phase means time normalized to stance phase, from heel contact to toe off

Table 2 Spatiotemporal parameters

\begin{tabular}{|c|c|c|c|c|}
\hline & No AFO & PFR1 & PFR2 & PFR3 \\
\hline Gait speed (m/s) & $0.76 \pm 0.01$ & $0.89 \pm 0.02$ & $0.92 \pm 0.04$ & $0.85 \pm 0.01$ \\
\hline Paretic side step length (m) & $0.43 \pm 0.01$ & $0.50 \pm 0.08$ & $0.53 \pm 0.05$ & $0.44 \pm 0.01$ \\
\hline Non paretic side step length (m) & $0.49 \pm 0.01$ & $0.49 \pm 0.04$ & $0.57 \pm 0.02$ & $0.48 \pm 0.03$ \\
\hline Cadence (steps/min) & $102.2 \pm 0.9$ & $101.1 \pm 0.7$ & $103.8 \pm 0.5$ & $107.8 \pm 2.9$ \\
\hline Step length ratio (non paretic/paretic) & $1.13 \pm 0.02$ & $0.99 \pm 0.08$ & $1.09 \pm 0.13$ & $1.10 \pm 0.09$ \\
\hline
\end{tabular}




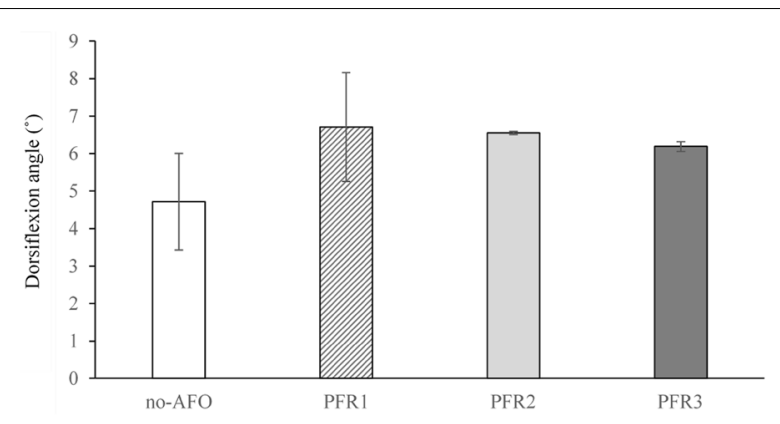

Fig. 4 Ankle joint angle in heel contact. The error bars represent \pm 1 standard deviation from the mean

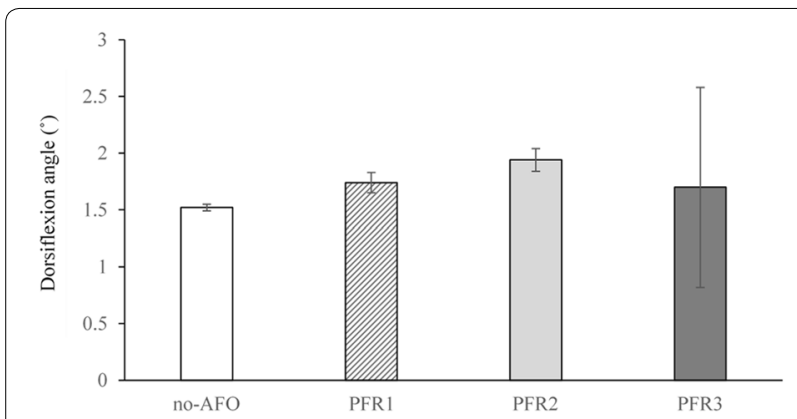

Fig. 5 Minimum dorsiflexion angle in early stance. The error bars represent \pm 1 standard deviation from the mean

Figure 5 shows the minimum dorsiflexion angle of the left side in the early stance phase. The minimum dorsiflexion angles in the early stance phase for each condition were: no AFO, $1.52^{\circ} \pm 0.03^{\circ}$; PFR $1,1.74^{\circ} \pm 0.09^{\circ}$; PFR2, $1.94^{\circ} \pm 0.10^{\circ}$; PFR3, $1.70^{\circ} \pm 0.88^{\circ}$. The minimum dorsiflexion angles were similar in all four conditions.

\section{Ankle and PFR torque}

Figure 6 shows the internal ankle joint torque of the left side in the stance phase, while Fig. 7 shows the peak internal dorsiflexion torque of the left side in the early stance phase. The peak internal dorsiflexion torques in the early stance phase for each condition were: no AFO, $6.48 \pm 3.95 \mathrm{Nm}$; PFR1, $14.3 \pm 2.39 \mathrm{Nm}$; PFR2, $8.62 \pm 3.51 \mathrm{Nm}$; PFR3, $9.96 \pm 1.01 \mathrm{Nm}$. The peak internal dorsiflexion torque values in all AFO conditions tended to be greater than that in the no AFO condition.

Figure 8 shows the PFR torque in the stance phase, while Fig. 9 shows the peak PFR torque in the stance phase. The peak PFR torque values in the stance phase for each condition were: PFR1, $1.83 \pm 0.05 \mathrm{Nm}$; PFR2, $2.35 \pm 0.06$; PFR3, $3.10 \pm 0.79 \mathrm{Nm}$.

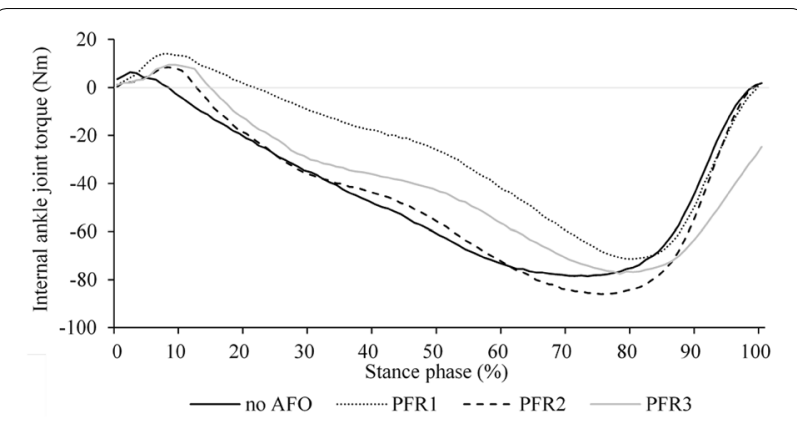

Fig. 6 Internal ankle joint torque in stance phase. Dorsiflexion is defined as positive. The $\%$ stance phase means time normalized to stance phase, from heel contact to toe off

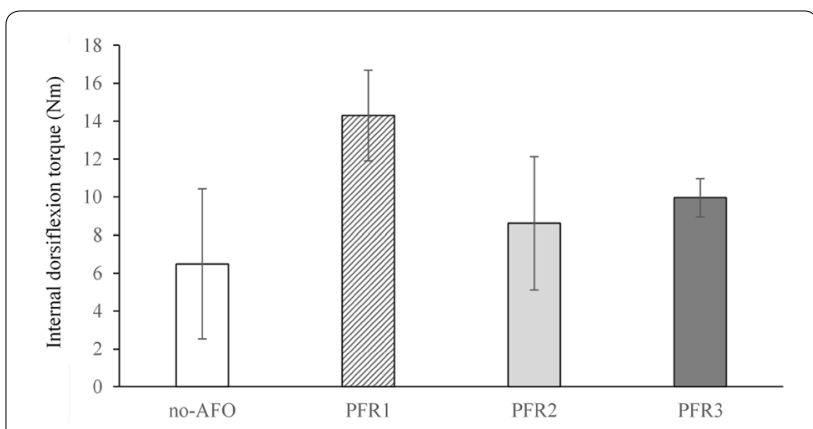

Fig. 7 Internal dorsiflexion torque in early stance. The error bars represent \pm 1 standard deviation from the mean

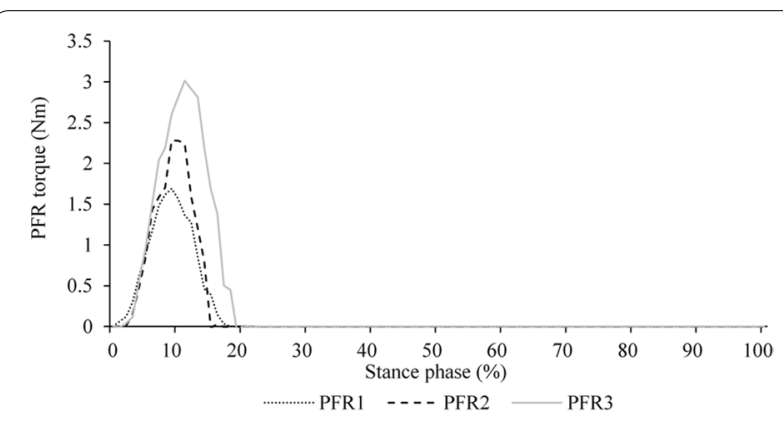

Fig. 8 PFR torque in stance phase. The \% stance phase means time normalized to stance phase, from heel contact to toe off

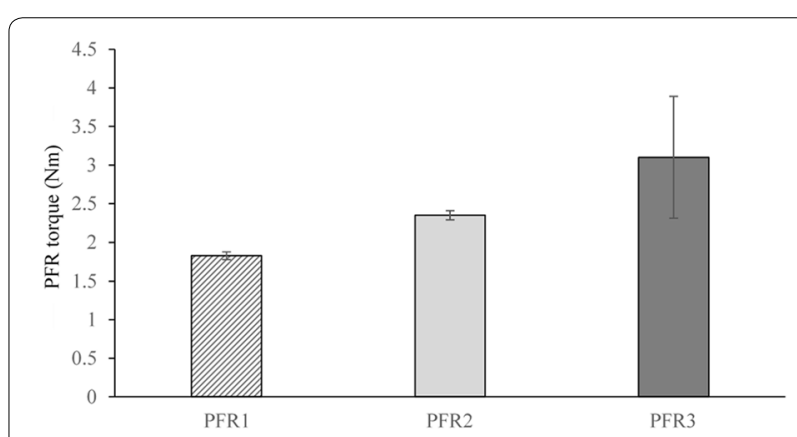

Fig. 9 Peak PFR torque in stance phase. The error bars represent \pm 1 standard deviation from the mean 


\section{Discussion}

The purpose of the present study was to investigate the effect of the magnitude of the PFR of an AFO on poststroke gait kinematics and kinetics during the early stance phase using a musculoskeletal model and an AFO model. Moreover, we investigated joint torque and PFR torque in stance phase separately.

Although the PFR function of the AFO also affects the swing phase, we focused on the early stance phase because this is the phase during which the PFR affects ankle function and weight acceptance [7, 8]; therefore, this function contributes to forward propulsion in the early stance phase.

The spatiotemporal parameters, especially gait speed and step length ratio, tended to improve under all three AFO conditions compared with the no AFO condition. These results are consistent with those reported in previous studies [4, 20]. Gait symmetry is also important for post-stroke gait function. The gait speed is decreased in people with chronic hemiparesis with an asymmetrical step length [20]. Moreover, an asymmetrical gait pattern increases the fall risk [21]. Although the present subject was able to walk independently without an AFO, the results show that using an AFO immediately improved his walking ability. The cadence increased for the PFR 3 condition, compared with other conditions. Although gait speed improved in PFR 3 condition, both step lengths were similar to no-AFO condition. Step length and cadence are major factor in determining gait speed. Therefore, increase of cadence seemingly compensated short step length.

Although the minimum dorsiflexion angle of the paretic side in the early stance phase was similar under all conditions, the ankle joint angle of the paretic side at heel contact was increased under all AFO conditions compared with the no AFO condition. Mulroy et al. [22] reported that the use of an AFO with a PFR function improves dorsiflexion at heel contact, while a rigid and plantar flexion stop type AFO prevents plantar flexion in the early stance phase. Although it is important for weight acceptance and forward propulsion to maintain the dorsiflexion angle at heel contact, most stroke patients find it difficult to maintain this position. In the present study, the minimum dorsiflexion angle was similar under all conditions. The PFR of the AFO used in the present study did not have a large degree of stiffness compared with a rigid AFO. Thus, all AFO conditions seemed to allow ankle movement as well as the no AFO condition.

The internal dorsiflexion torque of the paretic side in the early stance phase tended to increase more than the PFR torque. In the present simulations using the musculoskeletal model and the AFO model, the net ankle torque was computed by using inverse dynamics. Then, the ankle internal torque was computed by subtracting the PFR torque from the net torque. Therefore, this result shows purely the internal dorsiflexion torque. Dorsiflexion torque in the early stance phase is important for the first and second rocker functions, namely for the smooth ankle movement required for forward propulsion. An AFO with a PFR function assists in the performance of this function. The increase in the dorsiflexion torque was seemingly attributable to the improvements in the dorsiflexion angle and gait speed. Notably, the dorsiflexion torque was highest under the PFR1 condition. In clinical practice, the magnitude of the PFR of an AFO is customized to each patient's body function and gait performance. However, it is difficult to understand the relationship between the magnitude of the PFR and gait performance. The present simulation targeted a subject with post-stroke hemiplegia to evaluate the relationship between the magnitude of the PFR and the ankle torque, angle, and gait parameters.

The present study has one limitation. Although the present results indicated that the PFR of an AFO was related to gait performance after stroke, only one subject was evaluated. Further study of a large number of subjects with stroke is needed to confirm these findings.

\section{Conclusions}

The present study investigated the effect of the magnitude of the PFR of an AFO on post-stroke gait kinematics and kinetics during the early stance phase using a musculoskeletal model and an AFO model. Moreover, we investigated joint torque and PFR torque in stance phase separately. The simulation results showed that the PFR affects post-stroke gait kinematics, kinetics, and gait parameters such as gait speed and symmetry. The poststroke gait simulation in the present study contributes to the development of more effective gait rehabilitation treatment using an AFO.

\section{Abbreviations \\ AFO: ankle-foot orthosis; PFR: plantar flexion resistance. \\ Acknowledgements \\ We thank Kelly Zammit, BVSc, from Edanz Editing (http://www.edanzediti ng.com/ac), for editing a draft of this manuscript.}

\section{Authors' contributions}

In present study, MY performed musculoskeletal model and AFO model setting, measurements of subject walking, simulations, and data analysis. KS, $\mathrm{MH}$, and YK performed measurements and helped drafting this manuscript. All authors read and approved the final manuscript.

\section{Funding}

This research was supported by a research Grant from The Mazda Foundation. 
Availability of data and materials

All data generated or analyzed during this study are included in this published article.

\section{Ethics approval and consent to participate}

All procedures were approved by the ethics committee of the Faculty of Health and Welfare at Prefectural University of Hiroshima and were consistent with the Declaration of Helsinki. Written informed consent was obtained from the subject.

\section{Competing interests}

The authors declare that they have no competing interests.

\section{Author details}

${ }^{1}$ Graduate School of Engineering, Hiroshima University, 1-4-1, Kagamiyama, Higashi-Hiroshima City, Hiroshima 739-8527, Japan. ${ }^{2}$ Faculty of Health and Welfare, Prefectural University of Hiroshima, Hiroshima, Japan. ${ }^{3}$ JST, PRESTO, Hiroshima, Japan.

Received: 5 April 2019 Accepted: 11 July 2019

Published online: 16 July 2019

\section{References}

1. Wall JC, Turnbull GI (1986) Gait asymmetries in residual hemiplegia. Arch Phys Med Rehabil 67:550-553

2. Kim CM, Eng JJ (2004) Magnitude and pattern of 3D kinematic and kinetic gait profiles in persons with stroke: relationship to walking speed. Gait Posture 20:140-146

3. Hesse S, Werner C, Matthias K, Stephen K, Berteanu M (1999) Nonvelocity-related effects of a rigid double-stopped ankle-foot orthosis on gait and lower limb muscle activity of hemiparetic subjects with an equinovarus deformity. Stroke 30:1855-1861

4. Tyson SF, Kent RM (2013) Effects of an ankle-foot orthosis on balance and walking after stroke: a systematic review and pooled meta-analysis. Arch Phys Med Rehabil 94:1377-1385

5. Tyson SF, Sadeghi-Demneh E, Nester CJ (2013) A systematic review and meta-analysis of the effect of an ankle-foot orthosis on gait biomechanics after stroke. Clin Rehabil 27:879-891

6. Perry J, Burnfield JM (2010) Gait analysis: normal and pathological function. SLACK Incorporated, New York

7. Nolan KJ, Yarossi M (2011) Preservation of the first rocker is related to increases in gait speed in individuals with hemiplegia and AFO. Clin Biomech 26:655-660

8. Yamamoto S, Hagiwara A, Mizobe T, Yokoyama O, Yasui T (2009) Gait improvement of hemiplegic patients using an ankle-foot orthosis with assistance of heel rocker function. Prosthet Orthot Int 33:307-323

9. Murai A, Hobara H, Hashizume S, Kobayashi Y, Tada M (2018) Can forward dynamics simulation with simple model estimate complex phenomena?: case study on sprinting using running-specific prosthesis. Robomech J $5: 1-8$

10. Arch ES, Stanhope SJ, Higginson JS (2016) Passive-dynamic ankle-foot orthosis replicates soleus but not gastrocnemius muscle function during stance in gait: insights for orthosis prescription. Prosthet Orthot Int 40:606-616

11. Choi H, Peters KM, MacConnell MB, Ly KK, Eckert ES, Steele KM (2017) Impact of ankle foot orthosis stiffness on Achilles tendon and gastrocnemius function during unimpaired gait. J Biomech 64:145-152

12. Yamamoto M, Shimatani K, Hasegawa M, Murata T, Kurita Y (2018) Estimation of the knee joint reaction force based on the plantarflexion resistance of an ankle-foot orthosis during gait. J Phys Ther Sci 30:966-970

13. Yamamoto M, Shimatani K, Hasegawa M, Murata T, Kurita Y (2019) Effects of altering plantar flexion resistance of an ankle-foot orthosis on muscle force and kinematics during gait training. J Electromyogr Kinesiol 46:63-69

14. Yamamoto S, Hagiwara A, Mizobe T, Yokoyama O, Yasui T (2005) Development of an ankle-foot orthosis with an oil damper. Prosthet Orthot Int 29:209-219

15. Delp SL, Anderson FC, Arnold AS, Loan P, Habib A, John CT, Guendelman E, Thelen DG (2007) OpenSim: open-source software to create and analyze dynamic simulations of movement. IEEE Trans Biomed Eng 54:1940-1950

16. OpenSim Documentation. Simulation-based design to prevent ankle injuries. https://simtk-confluence.stanford.edu:8443/display/OpenSim/ Simulation-Based+Design+to+Prevent+Ankle+Injuries. Accessed 18 Mar 2019

17. Hamner SR, Seth A, Delp SL (2010) Muscle contributions to propulsion and support during running. J Biomech 43:2709-2716

18. OpenSim Documentation. How inverse dynamics works. https:// simtk-confluence.stanford.edu:8443/display/OpenSim33/How+Inver se+Dynamics+Works. Accessed 18 Mar 2019

19. van den Bogert AJ, Geijtenbeek T, Even-Zohar O, Steenbrink F, Hardin EC (2013) A real-time system for biomechanical analysis of human movement and muscle function. Med Biol Eng Comput 51:1069-1077

20. Bregman DJ, De Groot V, Van Diggele P, Meulman H, Houdijk H, Harlaar J (2010) Polypropylene ankle foot orthoses to overcome drop-foot gait in central neurological patients: a mechanical and functional evaluation. Prosthet Orthot Int 34:293-304

21. Wei TS, Liu PT, Chang LW, Liu SY (2017) Gait asymmetry, ankle spasticity, and depression as independent predictors of falls in ambulatory stroke patients. PLoS ONE 23:e0177136

22. Hall AL, Bowden MG, Kautz SA, Neptune RR (2012) Biomechanical variables related to walking performance 6-months following post-stroke rehabilitation. Clin Biomech 27:1017-1022

\section{Publisher's Note}

Springer Nature remains neutral with regard to jurisdictional claims in published maps and institutional affiliations.

\section{Submit your manuscript to a SpringerOpen ${ }^{\circ}$ journal and benefit from:}

- Convenient online submission

- Rigorous peer review

- Open access: articles freely available online

- High visibility within the field

Retaining the copyright to your article

Submit your next manuscript at springeropen.com 